\title{
Mengen'de Yetişen Bazı Yabani Mantar Türlerinin Doğal Boya Kaynağı Olarak Değerlendirilmesi
}

\author{
Filiz Yıldız ${ }^{*}$, Nigar Merdan², Habip Dayığlu ${ }^{3}$, Emine Dilara Koçak ${ }^{4}$ \\ 1*Bolu Abant İzzet Baysal Üniversitesi, Bolu Teknik Bilimler Meslek Yüksekokulu, Tekstil, Giyim, Ayakkabı ve Deri Bölümü, Bolu, Türkiye (ORCID: 0000-0001- \\ 7757-8888), akin_@@ibu.edu.tr \\ 2 İstanbul Ticaret Üniversitesi, Mimarlık ve Tasarım Fakültesi, Moda ve Tekstil Tasarımı Bölümü, İstanbul, Türkiye (ORCID: 0000-0001-7246-4849), \\ nmerdan@ticaret.edu.tr \\ ${ }^{3}$ İstanbul Ticaret Üniversitesi, Mimarlık ve Tasarım Fakültesi, Moda ve Tekstil Tasarımı Bölümü, İstanbul, Türkiye (ORCID: 0000-0002-2069-4412), \\ hdayioglu@ticaret.edu.tr \\ ${ }^{4}$ Marmara Ünivristesi, Teknoloji Fakültesi, Tekstil Mühendisliği Bölümü, İstanbul, Türkiye (ORCID: 0000-0002-0656-6430), dkocak@marmara.edu.tr
}

(İlk Geliş Tarihi 1 Temmuz 2020 ve Kabul Tarihi 12 Aralık 2020)

(DOI: 10.31590 /ejosat.762480)

ATIF/REFERENCE: Yıldız, F. Merdan, N. Dayığlu, H. \& Koçak D.E. (2020). Mengen'de Yetişen Bazı Yabani Mantar Türlerinin Doğal Boya Kaynağı Olarak Değerlendirilmesi. Avrupa Bilim ve Teknoloji Dergisi, (20), 751-759.

$\ddot{O} \mathbf{z}$

Bu çalı̧̧mada, doğal boya olarak chantarelle (Cantharellus Cibarius), porcini (Boletus Edulis ) ve trumpet (Craterellus Tubaeformis) yabani mantarların ekstraksiyon ürünleri kullanılmıştır. Cantharellus Cibarius, Boletus Edulis ve Craterellus Tubaeformis türlerinden mantarlar Bolu Mengen ilçesinden toplanmış, yıkandıktan sonra etüvde $\left(40^{\circ} \mathrm{C} \pm 2^{\circ} \mathrm{C}\right) 8$ saat kurutulmuştur. Materyal olarak 160 denye $\% 100$ ipek iplik kullanılmıştır. Boyamalarda kullanılacak ipek iplikler laboratuvar tipi boyama makinesinde (Termal) $20 \mathrm{~g} / \mathrm{l}$ zeytinyağ sabun çözeltisi 1 saat kaynatılmış ve ardından materyale sıcak ve soğuk yıkama işlemleri yapılarak materyaller kurutulmuştur. Boyamadan önce ipek iplikler bakır sülfat, potasyum alüminyum sülfat, potasyum dikromat, kalay (II) klorür, demir (II) klorür ve demir (III) klorür ile mordanlanmıştır. Çektirme yöntemine göre, iplikler laboratuvar boyama makinesinde 60 dakika kaynama sicaklığında boyanmış, flotte içerisinde soğutulmaya bırakılarak 12 saat sonra örneklere, soğuk taşar yıkama, sıcak yıkama, kaynar sabunlama ve soğuk durulama işlemleri yapılmıştır. Kurutma işleminden sonra renk koordinatları oluşturmak için spektrofotometre (Datacolor Spectra Flash 600 plus- D65 $\left(10^{\circ}\right.$ ) ile renk ölçümleri gerçekleştirilmiştir. Boyanmış örneklerin yıkama, sürtme ve ışık haslıkları incelenmiştir. Sonuç olarak: Mordan maddelerinin, toplam renk farklılığı $\left(\Delta \mathrm{E}^{*}\right)$ değişiminde etkili olduğu belirlenmiş ve en yüksek toplam renk farklılık değeri, bakırsülfat mordanının kullanıldığı boyamada elde edilmiştir. $\left(\Delta \mathrm{E}^{*}=10.2884\right)$. Açıklık - koyuluk $\left(\Delta \mathrm{L}^{*}\right)$ eksenine göre renk değerlendirilmesinde, potasyum bikromat ile mordanlanmış örnek dışında, diğer boyamalarda renk standarttan daha açık elde edilmiştir.

Chantarelle, porcini ve trumpet mantarlarlarından elde edilen boyarmaddeler ile ipekli materyalin boyanmasında Renk Kuvveti (K/S) değerleri karşılaştırıldığında; en iyi renk veriminin demir (II) klorür ile mordanlandıktan sonra porcini mantarından elde edilen doğal boya ile boyanmış ipek materyalde elde edildiği görülmektedir. Mordansız boyama ve demir (III) klorür, potasyum dikromat ve kalay klorür mordanları ile yapılan tüm boyamalarda renk verimi oldukça düşüktür.

Porcini mantarından elde edilmiş doğal boya ile boyanmış örneklerde, en yüksek toplam renk farklılık değerinin, demir (II) klorür mordanının kullanıldığı boyamada elde edildiği görülmüştür. Trumpet mantarından elde edilen doğal boya ile boyanmış ipek örneklerinde; en yüksek toplam renk farklılık değeri, bakır sülfat mordanının kullanıldığı boyamada elde edilmiştir.

Anahtar Kelimeler: Doğal Boya, Yabani Mantar, İpek İplik, Renk Özellikleri.

*Filiz YILDIZ: akin_f@ibu.edu.tr 


\title{
Evaluation of Some Wild Mushroom Species Growing in Mengen as a Natural Dye Source
}

\begin{abstract}
In this study, extraction products of chantarelle (Cantharellus Cibarius), porcini (Boletus Edulis) and trumpet (Craterellus Tubaeformis) wild mushrooms were used as a natural dyes. Cantharellus Cibarius, Boletus Edulis and Craterellus Tubaeformis mushroom species were collected from Bolu Mengen district, washed and then dried in the oven $\left(40^{\circ} \mathrm{C} \pm 2{ }^{\circ} \mathrm{C}\right)$ for 8 hours. $100 \%$ silk treat with 160 denye was used as a material to be dyed. The silk treads to be used in dyeing, were boiled in a laboratory type dyeing machine (Emsey Teknik) by applying $20 \mathrm{~g} / \mathrm{l}$ olive oil soap solution for 60 minutes, and then the material was dried by performing hot and cold water washing processes. Before dyeing, silk treads were mordanted with copper sulphate, potassium aluminum sulphate, potassiun dichromate, tin(II)chloride, iron(II)chloride and iron(III)chloride to see the effects of different mordants. According to the exhaustion method, the silk yarns were were dyed in the laboratory dyeing machine for 60 minutes at boiling temperature (around $98^{\circ} \mathrm{C}$ ), then left to cool in the dyeing liquor, finally followed cold overflow washing, hot washing and cold rinsing additionally. After drying the samples, color measurements were carried out with a spectrophotometer (Datacolor Spectra Flash 600 Plus-D65/10 ${ }^{\circ}$ ) to create color coordinates. Color fastness to washing, rubbing and light of the dyed samples were examined. As a result, it was determined that mordant substances were effective in the change of total color difference $(\Delta \mathrm{E})$ and the highest value was obtained in the dyeing using copper suphate mordant $(\Delta \mathrm{E} 10.2884)$. In the evaluation of the color according to the lightness-darkness $(\Delta \mathrm{L})$ axis, the color was obtained lighter than the standard in other dyeings, except for the sample that was mordanted with potassium dichromate.

Comparing the color strength $(\mathrm{K} / \mathrm{S})$ values of the dyestuffs obtained from chantarelle, porcini and trumpet mushrooms on the dyed silk, it was observed that the best color yield was obtained in the silk material dyed with natural dye obtained from porcini mushroom after being mordanted with iron (II) chloride. The color yield was very low in the dyeing without mordant and all dyeing with iron (III) chloride, potassium dichromate and tin chloride mordants.

In the samples dyed with natural dyes obtained from porcini mushroom, the highest total color difference value was obtained in the dyeing using iron(II)chloride mordant. Silk samples dyed with natural dye obtained from trumpet mushroom, the highest total color difference value achieved in the dyeing using copper suphate mordant.
\end{abstract}

Keywords: Natural Dye, Wild Mushroom, Silk Yarn, Colour Properties.

\section{Giriş}

Doğal boyarmaddelerin tekstil liflerinde kullanılması Hindistan ve Mezopotamya'da MÖ 4000 yıllarında başlamış, sentetik boyarmaddelerin sentezlenerek üretilmesine kadar önemini koruyarak 19. yüzyıl sonlarında önemini yitirmiştir. Sentetik boyarmaddelerin, çevreye vermiş olduğu zararlar ve alerjiye sebep olması gibi nedenlerle günümüzde doğal boyalara olan ilgi artmıştır (Santis ve Moresi, 2007). Bitkilerden, hayvanlardan, bakterilerden ve mantarlardan türetilen doğal boyaların biyo-bozunurluğu ve düşük alerjik reaksiyonları nedeniyle sentetik renklendiricilere biyo-uyumlu, güvenli ve yenilenebilir alternatifler olduğuna inanılmaktadır (Rather ve ark., 2016).

Doğal boya kaynağı olarak kullanılan birçok bitkinin renklilik ve haslık özelliklerinin incelendiği çalışmalar literatürde yer almaktadır (Mirjalili ve ark., 2011; Lee ve Kim 2004; Priti ve ark., 2013) . İpeğin doğal boyarmaddelerle renklendirilmesinde, çok çeşitli mordan maddeleri kullanılmış ve bu maddelerin boyamaların renklilik özelliğine etkileri incelenmiştir (Vankar ve ark. 2007; Prabhu ve ark. 2011). Lifleri katyonikleştirme (Nakpathom ve ark., 2018), plazma ve fikse maddeleri ile ön işlem (Dayioglu ve ark., 2015), ultrasonik enerji ve ozonla ön işlem ( Benli ve Bahtiyari, 2015), gama ışınlarının boyamaya etkisi (Bhatti ve ark., 2010) gibi doğal boyanın liflere ilgisini artırmak için bazı teknikler de geliştirilmiştir.

Birçok doğal boya bitkisinin antioksidan ve UV koruma özellikleri vardır. Antioksidan etkiden sorumlu yapıların fenolik asitler, flavonoidler, tokoferoller gibi fenolik gruplar olduğu bilinmektedir (Prusty, A.K. ve ark. 2010; Wang ve ark. 2009; Mongkholrattanasit ve ark. 2011; Mongkholrattanasit ve ark. 2011). Araştırmacılar, doğal boyarmaddelerin uygulamalarına ait ultrasonik enerji, mikrodalga enerjisi ve süperkritik karbondioksit ile boyama gibi birçok yeni yöntem geliştirmişlerdir (Vankar ve Shanker,2006; Elshemy 2011; Liu ve ark. 2018; Shahid ve ark. 2013).

$\mathrm{Bu}$ araştırmada, chantarelle (Cantharellus Cibarius), porcini (Boletus Edulis) ve trumpet (Craterellus Tubaeformis ) yabani zehirli olmayan mantar ekstraktları ile boyanmış ipekli materyalin kolorimetrik ve haslık özellikleri incelenmiştir. Literatürde farklı kaynaklardan elde edilmiş doğal boyaların ipek ve diğer tekstillerin renklendirilmesinde kullanımı ile ilgili çok sayıda çalışma mevcut olmasına karşın, mantarların doğal boya olarak kullanıldığı çalışmalar daha azdır. Dermocybe Sanguinea mantarından antrakinon pigmentlerinin izolasyonu için basit bir enzimatik yöntem geliştirilerek glukozit formundaki emodin ve dermosinbin pigmentleri elde edilmiştir ( Hynninen ve ark., 2000). Sarcodon Imbricatus ( Rice ve Beebee, 1980), Hydnellum Peckii (Bessete ve Bessete, 2001), Phaeolus Schweinitzii 
(Räisänen R. 2002), Laetiporus Sulphureus (Weber ve ark., 2004), Ganoderma Applanatum, Coriolus Versicolor ve Amanita Muscaria ( Perumal, 2004) çeşitli liken ve mantarlarda bulunan pigmentlerin kimyasal yapıları ( Raisanen, 2009), mantarlardan elde edilen doğal boyaların pamuk ve ipek kumaşlara uygulanabilirliği ( Karuppan ve ark., 2014), bazı mantarlardaki doğal antrokinon emodin ve dermocybin yapılarının poliester, poliamid ve yün boyamadaki renk ve haslık özellikleri ( Raisanen ve ark., 2001; Raisanen ve ark., 2001), Pycnoporus Sanguineus mantarından mikro dalga enerjisi kullanılarak ekstrakte edilen doğal boyanın ipekli materyal için renk ve haslık özellikleri incelenmiştir (Ahmad ve ark., 2014). Mantarların tedavi etme ve boya üretim özellikleri, (Tidke ve ark., 2006), chaga mantar ekstraktına (Inonotus obliquus) uygulanan gama ışınlarının, renk ve antioksidan özelliklerini iyileştirdiği (Kim ve ark., 2009), Chanterelle Mushroom ve Cantharellus cibarius mantarlarının antioksidan aktivitesinin incelendiği (Ebrahimzadeh ve ark., 2015), Chanterelle ve Cantharellus cibarius mantarlarının iltihap tedavisi ve yara iyileştirici özellikleri (Nasiry ve ark., 2017), yabani mantar ekstrelerindeki toplam fenolik içeriği ile antioksidan aktiviteleri arasında ilişkinin incelendiği (Cheung ve ark., 2003) çalışmalar da literatürde yer almaktadır. Ayrıca literatür de yenilebilir bir mantar türü olan Pleurotus ostreatus'un, sulu çözeltilerden Cr (VI) iyonlarının biyosorpsiyonu için biyosorbent olarak kullanılabilirliği üzerine çalışmada mevcuttur (Sarıkaya, 2019).

$\mathrm{Bu}$ çalışmada, doğal boya olarak chantarelle (Cantharellus Cibarius), porcini (Boletus Edulis) ve trumpet (Craterellus Tubaeformis) yabani mantarların ekstraksiyon ürünleri kullanılmıştır. Cantharellus Cibarius, Boletus Edulis ve Craterellus Tubaeformis türlerinden mantarlar Bolu Mengen ilçesinden toplanmış, y1kandıktan sonra etüvde $\left(40^{\circ} \mathrm{C} \pm 2{ }^{\circ} \mathrm{C}\right) 8$ saat kurutulmuştur. Materyal olarak 160 denyede \% 100 ipek iplik kullanılmıştır. Boyamalarda kullanılacak ipek iplikler laboratuvar tipi boyama makinesinde (Termal) $20 \mathrm{~g} / \mathrm{l}$ zeytinyağı sabun çözeltisi ile 1 saat kaynatılmış ve ardından materyale sıcak ve soğuk yıkama işlemleri yapılarak materyaller kurutulmuştur. Boyamadan önce ipek iplikler bakır sülfat, potasyum alüminyum sülfat, potasyum dikromat, kalay (II) klorür, demir (II) klorür ve demir (III) klorür ile mordanlanmıştır. Çektirme yöntemine göre iplikler, laboratuvar boyama makinesinde 60 dakika kaynama sicaklığında boyanmış, flotte içerisinde soğutulmaya bırakılmıştır. 12 saat sonra örneklere, soğuk taşar yıkama, sıcak yıkama, kaynar sabunlama ve soğuk durulama işlemleri yapılmıştır. Kurutma işleminden sonra renk koordinatları oluşturmak için spektrofotometre (Datacolor Spectra Flash 600 plus- D65/ $10^{\circ}$ ) ile renk ölçümleri gerçekleştirilmiştir. Boyanmış örneklerin yıkama, sürtme ve 1şık haslıklarına bakılmıştır.

\section{Materyal ve Metot}

\subsection{Materyal}

Bu çalışmada materyal olarak 160 denye iplik numarasında \% 100 ipek iplik kullanılmıştır. Çalışmada kullanılan mantarlar (Cantharellus Cibarius, Boletus Edulis ve Craterellus Tubaeformis) Bolu ili Mengen ilçesi ormanlarından elde edilmiştir. Çalışma da doğal boya olarak kullanılan mantarlar Şekil 1'de verilmiştir. Doğal boyarmadde olarak kullanılan mantarlar yapılarında Melanin, Karotenoid, Emodin, Dermocybin pigmentlerini içermektedir ( Şekil 2).

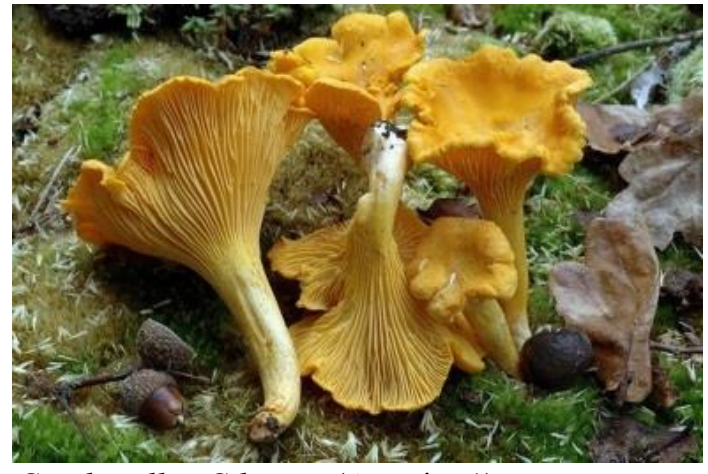

Cantharellus Cibarius.(Anonim 1)

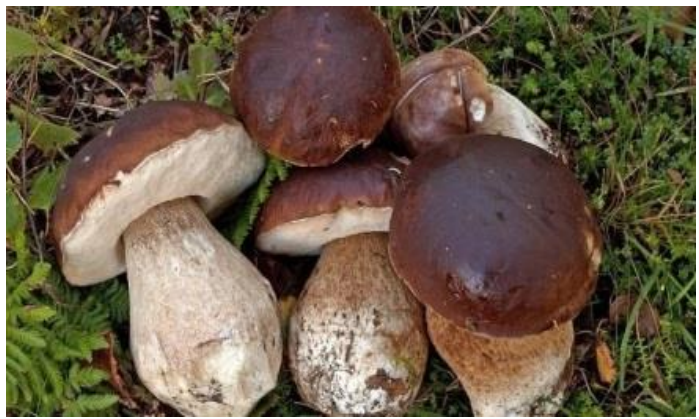

Boletus edulis Bull.(Anonim 2)

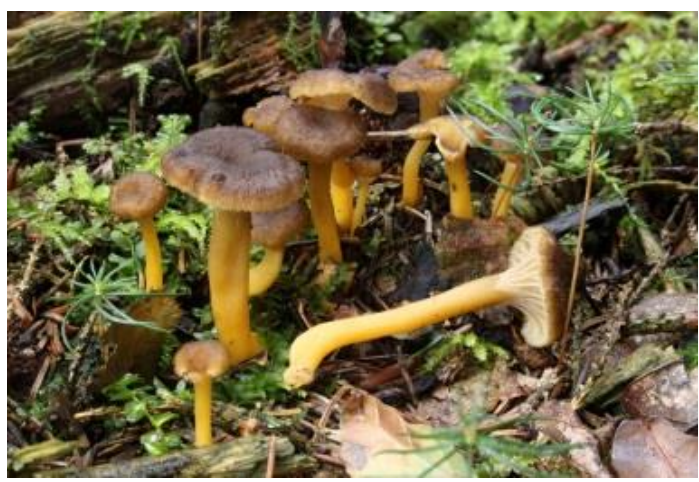

Craterellus tubaeformis (Anonim 3)

Şekil 1. Doğal Boya Kaynağı olarak Kullanılan Mantarlar<smiles></smiles>

Melanin<smiles>Cc1cc(O)c2c(c1)C(=O)c1cc(O)cc(O)c1C2=O</smiles>

Emodin<smiles>CC(C)=CC=C(C)C=CC=C(C)C=CC=CC(C)=CC=CC(C)=CC=CC1=C(C)C(=O)CCC1(C)C</smiles>

Karotenoid<smiles>COc1c(O)c(O)c2c(c1O)C(=O)c1cc(C)cc(O)c1C2=O</smiles>

Dermocybin

Şekil 2. Bazı Mantarlardaki Pigmentler 


\subsection{Metod}

\subsection{1. İpeğin Serisininin Uzaklaştırılması}

Boyamalarda kullanılacak 160 denyede 2 g ipek iplik, laboratuvar tipi boyama makinesinde (Termal) $20 \mathrm{~g} / 1$ zeytinyağ sabun çözeltisi ile $\mathrm{pH} 8$ 'de, 1/50 flotte oranındaki flottede 1 saat kaynama sıcaklığında çalışılmıştır. Ardından flotte oranı 1/50 olan flottelerde, ilk yıkama $60{ }^{\circ} \mathrm{C}$ ' de 15 dakika, ikinci yıkama 40 ${ }^{\circ} \mathrm{C}$ 'de 15 dakika ve son yıkamada oda sıcaklığında olmak üzere yıkanmıştır ve ipek iplik numuneleri etüv de $105^{\circ} \mathrm{C}$ 'de 2 dakika kurutulmuştur.

\subsubsection{Boyarmadde Ekstraksiyonu}

Cantharellus Cibarius, Boletus Edulis ve Craterellus Tubaeformis mantarları destile suda, oda sıcaklığında $\left(20{ }^{0} \mathrm{C}\right)$ yıkandiktan sonra etüvde $\left(40^{\circ} \mathrm{C} \pm 2^{\circ} \mathrm{C}\right) 8$ saat kurutulmuştur. Daha sonra $40 \mathrm{~g}$ kurutulmuş mantar, flotte oranı $1 / 20$ olacak şekilde 60 dakika oda sıcaklığında bekletildikten sonra, manyenik karıştırıcı yardımı ile kaynama sıcaklığında 60 dakika kaynatılmış, süzülerek boyamaya hazır duruma getirilmiştir.

\subsubsection{Mordanlama}

Liflerin doğal boyalara karşı ilgisi fazla olmadığı için mordanlama işlemi yapılır. Metal mordanlar, tekstil materyallerinin doğal boya ile boyanmasında en çok tercih edilen maddelerdir. Bu maddeler boya ile lif arasında lif - mordan - boya kompleksini oluştururlar (Şekil 3).

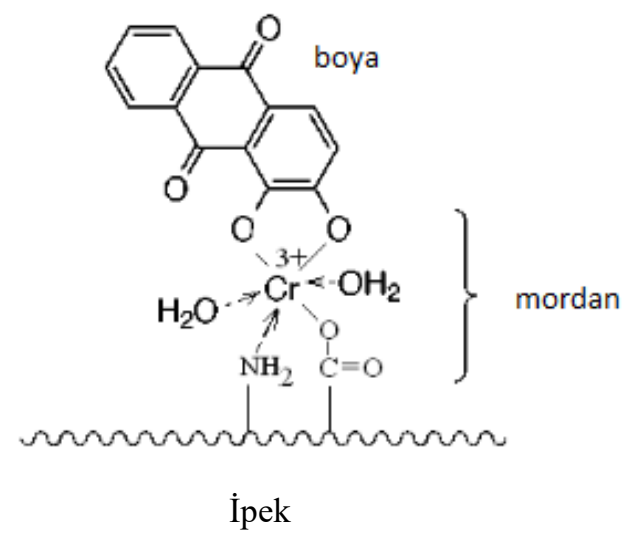

Şekil 3. Mordan Yardımı ile Boya - Metal Kompleks Oluşumu

$\mathrm{Bu}$ çalışmada ipek örneklerinin mordanlanmasında ön mordanlama yöntemi kullanılmıştır. Mordanlama işleminde bakır sülfat, potasyum alüminyum sülfat, potasyum dikromat, kalay (II) klorür, demir (II) klorür ve demir (III) klorür olmak üzere 6 farklı mordan kullanılmıştır.

Mordanlama işleminde flotte oranı 1/40 olacak şekilde ayarlanmış ve bütün mordanlar için $\% 1$ mordan maddesi (materyal ağırlığına göre) kullanılarak, laboratuvar boyama makinesinde, 60 dakika kaynama sıcaklığında çalışılmış ve materyallerdeki fazla çözelti uzaklaştırılarak kendi halinde kurutulmaya bırakılmıştır.

\subsection{4. İpek İpliklerinin Boyanması}

Boyamalar çektirme yöntemine göre yapılmış ve elde edilen boya ekstraktları seyreltilmeden kullanılmıştır. Şekil 4'de verilen sıcaklık-zaman diyagramına göre $2 \mathrm{~g}$ ağırlıktaki ipek iplik örnekleri, flotte oranı 1/40 olan flottede boyanmıştır. Daha sonra flotte içerisinde 12 saat soğutulmaya bırakılmış ve örneklere soğuk taşar yıkama $(250 \mathrm{ml}), 60^{\circ} \mathrm{C}$ sıcak y1kama (100ml), kaynar sabunlama ( $1 \mathrm{~g} / \mathrm{l}$ noniyonik deterjan $100 \mathrm{ml}$ ) ve soğuk durulama $(100 \mathrm{ml})$ işlemleri yapılmıştır. Örnekler etüvde $105^{\circ} \mathrm{C}$ 'de 2 dakika kurutulmuştur.

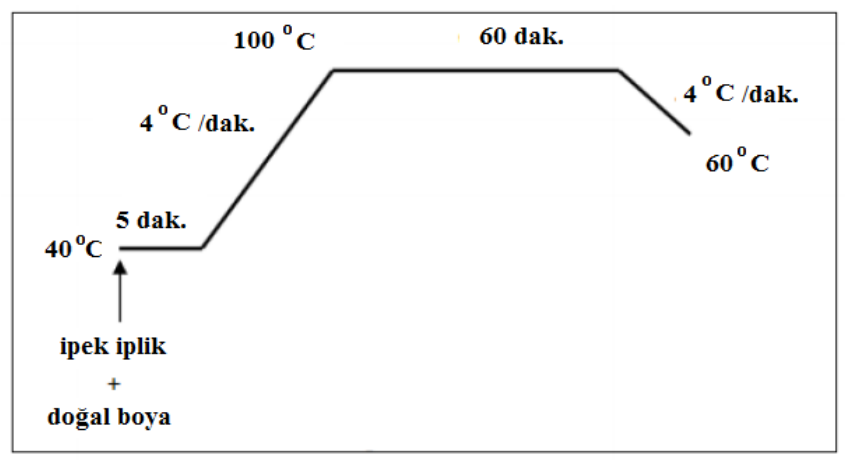

Şekil 4. Boyamaların Sıcaklık-Zaman Diyagramı

\subsection{4. Ölçüm Metotları}

Çalışmada elde edilen boyama örneklerinin toplam renk farklılıkları, renk kuvvetleri ve renk haslık değerleri belirlenmiş ve aşağıda açıklanmıştır.

\subsubsection{Renk Ölçümleri}

Renk ölçümleri için Datacolor SF 600 renk ölçüm cihazı ve Datamaster bilgisayar programı kullanılmıştır. CIELab sistemine göre, D65 illuminatı altında $10^{\circ}$ standart gözlemci kullanılarak gerçekleştirilen ölçümlerde, mordanlanmadan boyanmış örnek standart olarak kabul edilmiştir. Renklerin $\Delta E^{*}, \Delta L^{*}, \Delta a^{*}, \Delta b^{*}$, $\Delta C^{*}$ ve $\Delta H^{*}$ değerleri aşağıda verilen denklem 1 ile hesaplanmıştır.

$\Delta E^{*}=\sqrt{\left.\left[\left(\Delta L^{*}\right)^{2}+\left(\Delta a^{*}\right)^{2}+\left(\Delta b^{*}\right)^{2}\right)\right]}$

Burada; $\Delta L^{*}$ açıklık - koyuluk, $a^{*}$ kırmızılık - yeşillik, $b^{*}$ sarılık - mavilik, $\Delta E^{*}$ toplam renk farklılığını ifade etmektedir.

Boyanmış örneklerin renk kuvveti değerleri denklem 2'de verilen (K/S) Kubelka-Munk eşitliği kullanılarak hesaplanmıştır.

$K / S=(1-R)^{2} / 2 R$

Burada, $K$ absorbsiyon katsayısını, $S$ saçınım katsayısını, $R$ maksimum absorbsiyondaki dalga boyunda lifin reflektans değerini ve $K / S$ ise renk kuvvetini ifade etmektedir.

\subsubsection{Renk Haslık Ölçümleri}

Boyanmış örneklerin yıkama haslıkları, yıkama haslık test cihazında (Gyrowash-James H. Heal) ISO 105-C06 standartlarına uygun olarak yapılmıştır. Boyanmış olan numuneler $4 \mathrm{~g} / \mathrm{l}$ olacak şekilde hazırlanmış olan ECE deterjanı çözeltisinden 1/100 flotte oranında $40{ }^{\circ} \mathrm{C}$ sicaklıkta, 30 dakika Gyrowash Washer Tester Cihazında işlem görmüş ve gri skala ile değerlendirilmiştir.

Boyanmış olan ipek ipliklerin sürtme haslık testleri, Crockmeter Test cihazında ISO 105-X12:2016 'ya göre test edilmiş ve gri skala ile yaş ve kuru lekeleme haslık değerleri belirlenmiştir. Boyamaların 1şık haslıkları ise 1şık haslık test cihazında (Atlas Alfa 150 S) EN ISO 105 - B02-1994 standardına göre gerçekleştirilmiş ve renk değişimi mavi skala ile değerlendirilmiştir. 


\section{Araştırma Sonuçları ve Tartışma}

$\mathrm{Bu}$ bölümde araştırma sonuçlarında elde edilen sonuçlar değerlendirilmiştir.

\subsection{Renklerin Subjektif Değerlendirilmesi}

Serisini uzaklaştırılmış, mordansız ve 6 farklı mordan maddesi ile mordanlandıktan sonra boyanmış ipek ipliklerinin renk görüntüleri Tablo 1 de verilmiştir.

\section{Tablo 1. Boyanmış Ipek İpliklerin Renk Görüntüleri}

\begin{tabular}{c|c|c|c}
\hline Adı & Chantarelle & Porcini & Trumpet \\
\hline Mordansiz & & & \\
\hline $\mathrm{CuSO}_{4}$ & & & \\
& & & \\
\hline $\left.\mathrm{KAl}_{2} \mathrm{SO}_{4}\right)$ & & & \\
\hline $\mathrm{FeCl}_{2}$ & & & \\
\hline $\mathrm{K}_{2} \mathrm{Cr}_{2} \mathrm{O}_{7}$ & & & \\
& & & \\
\hline $\mathrm{SnCl}_{2}$ & & & \\
& & & \\
\hline
\end{tabular}

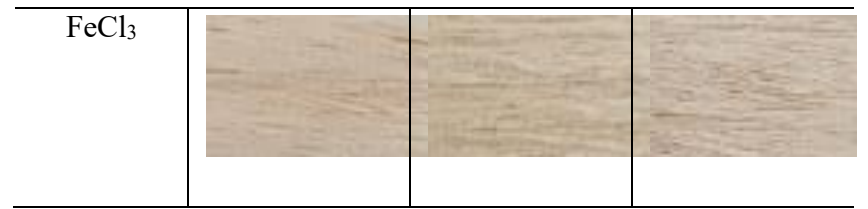

\subsection{Renk Ölçüm Sonuçları}

\subsection{1. $\Delta E^{*}, \Delta L^{*}, \Delta a^{*}, \Delta b^{*}$ ve $\Delta C^{*}$ Değerleri}

CIELab sistemine göre, D65 illuminatı altında $10^{\circ}$ standart gözlemci kullanılarak gerçekleştirilen ölçümlerde, mordanlanmadan boyanmış örnek standart olarak kabul edilerek denklem 1'e göre $\Delta \mathrm{E}^{*}, \Delta \mathrm{L}^{*}, \Delta \mathrm{a}^{*}, \Delta \mathrm{b}^{*}$ ve $\Delta \mathrm{C}^{*}$ değerleri hesaplanmıştır. Tablo 2'de Chantarelle (Cantharellus Cibarius) mantarından elde edilen doğal boya ile boyanmış örneklerin $\Delta \mathrm{E}^{*}, \Delta \mathrm{L}^{*}, \Delta \mathrm{a}^{*}, \Delta \mathrm{b}^{*}$ ve $\Delta \mathrm{C}^{*}$ değerleri yer almaktadır.

Chantarelle (Cantharellus Cibarius) mantarından elde edilen doğal boya ile boyanmış örneklerin $\Delta \mathrm{E}^{*}, \Delta \mathrm{L}^{*}, \Delta \mathrm{a}^{*}, \Delta \mathrm{b}^{*}$ ve $\Delta \mathrm{C}^{*}$ değerleri Tablo 2'de verilmiştir.

Altı farklı mordan maddesi ile mordanlama yapıldıktan sonra, chantarelle mantarından elde edilen doğal boya ile boyanmış ipek örneğinde, mordan maddelerinin toplam renk farklılığg $\left(\Delta \mathrm{E}^{*}\right)$ değişiminde etkili olduğu belirlenmiştir. Mordansız boyamaların standart olarak kabul edildiği renk ölçümlerinde en yüksek toplam renk farklılık değeri, bakırsülfat mordanının kullanıldığı boyamada elde edilmiştir $\left(\Delta \mathrm{E}^{*}=10.2884\right)$. Açıklık - koyuluk $\left(\Delta \mathrm{L}^{*}\right)$ eksenine göre renk değerlendirilmesinde, potasyum bikromat ile mordanlanmış örnek dışında, diğer boyamalarda renk standarttan daha açık elde edilmiştir. Kırmızı- yeşil $\left(\Delta \mathrm{a}^{*}\right)$ ekseninde, potasyum alüminyum sülfat ve kalay(II)klorürün kullanıldığı boyamalarda renk, kırmızı nüansa kaymıştır. Mavisarı ekseninde $\left(\Delta b^{*}\right)$ ise, sadece potasyum alüminyum sülfat mordanlı boyamada renk, sarı nüanslıdır. Boyamaların genellikle doygunluk değeri $\left(\Delta C^{*}\right)$ düşük ve renkler mattır (Tablo 2).

Tablo 2. Chantarelle (Cantharellus Cibarius) Mantarından Elde Edilen Doğal Boya ile Boyanmış Örneklerin $\Delta E^{*}, \Delta L^{*}, \Delta a^{*}, \Delta b^{*}$ ve $\triangle C^{*}$ Değerleri

\begin{tabular}{l|c|c|c|c|c}
\hline \multicolumn{1}{c|}{ Adı } & $\Delta E^{*}$ & $\Delta L^{*}$ & $\Delta a^{*}$ & $\Delta b^{*}$ & $\Delta C^{*}$ \\
\hline $\mathrm{CuSO}_{4}$ & 10.2884 & 9,780 & $-0,420$ & $-3,060$ & $-3,080$ \\
\hline $\mathrm{KAl}_{2}\left(\mathrm{SO}_{4}\right)_{2}$ & 0,17059 & 0,079 & 0,150 & 0,019 & 0,040 \\
\hline $\mathrm{K}_{2} \mathrm{Cr}_{2} \mathrm{O}_{7}$ & 1,340 & $-1,340$ & $-0,010$ & $-0,009$ & $-0,009$ \\
\hline $\mathrm{SnCl}_{2}$ & 1,1737 & 1,010 & 0,560 & $-0,210$ & $-0,179$ \\
\hline $\mathrm{FeCl}_{2}$ & 6,9329 & 4,269 & $-1,850$ & $-5,140$ & $-5,34$ \\
\hline $\mathrm{FeCl}_{3}$ & 4,2574 & 2,629 & $-1,449$ & $-3,019$ & $-3,179$ \\
\hline
\end{tabular}


Porcini (Boletus Edulis) mantarından elde edilen doğal boya ile boyanmış örneklerin $\Delta \mathrm{E}^{*}, \Delta \mathrm{L}^{*}, \Delta \mathrm{a}^{*}, \Delta \mathrm{b}^{*}$ ve $\Delta \mathrm{C}^{*}$ değerleri Tablo 3 'te verilmiştir.

Porcini mantarından elde edilmiş doğal boya ile boyanmış örneklerde, en yüksek toplam renk farklılık değerinin, demir (II) klorür mordanının kullanıldığı boyamada elde edildiği görülmüştür $\left(\Delta \mathrm{E}^{*}=6,8967\right)$. Potasyum alüminyum sülfat, potasyum dikromat ve kalay (II) klorürün mordan olarak kullanıldığı boyamalar standarttan daha koyudur. Bakır sülfat, kalay klorür ve demir (II) klorürde, renk maviye kayarken, doygunluk azalmış, potasyum alüminyum sülfat, potasyum dikromat ve demir (III) klorürde ise renk sarıya doğru giderken doygunluk yani kroma artmıştır. Kırmızı - yeşil ekseninde boyamalar genellikle kırmızı yönündedir (Tablo 3).

Tablo 3. Porcini (Boletus Edulis) Mantarından Elde Edilen Doğal Boya ile Boyanmış Örneklerin $\Delta E^{*}, \Delta L^{*}, \Delta a^{*}, \Delta b^{*}$ ve $\Delta C^{*}$ Değerleri

\begin{tabular}{l|c|c|c|c|c}
\hline Adı & $\Delta \boldsymbol{E}^{*}$ & $\Delta \boldsymbol{L}^{*}$ & $\Delta \boldsymbol{a}^{*}$ & $\Delta \boldsymbol{b}^{*}$ & $\Delta \boldsymbol{C}^{*}$ \\
\hline $\mathrm{CuSO}_{4}$ & 4,3527 & 4,08 & 0,41 & $-1,46$ & $-1,43$ \\
\hline $\mathrm{KAl}\left(\mathrm{SO}_{4}\right)_{2}$ & 1,5536 & $-1,40$ & 0,44 & 0,51 & 0,55 \\
\hline $\mathrm{K}_{2} \mathrm{Cr}_{2} \mathrm{O}_{7}$ & 3,3501 & $-2,3$ & 0,17 & 2,43 & 2,43 \\
\hline $\mathrm{SnCl}_{2}$ & 3,0927 & $-1,56$ & $-0,05$ & $-2,67$ & $-2,66$ \\
\hline $\mathrm{FeCl}_{2}$ & 6,8967 & 6,61 & $-0,33$ & $-1,94$ & $-1,97$ \\
\hline $\mathrm{FeCl}_{3}$ & 2,5162 & 1,6 & 0,09 & 1,94 & 1,93 \\
\hline
\end{tabular}

Trumpet (Craterellus Tubaeformis) mantarından elde edilen doğal boya ile boyanmış örneklerin $\Delta \mathrm{E}^{*}, \Delta \mathrm{L}^{*}, \Delta \mathrm{a}^{*}, \Delta \mathrm{b}^{*}$ ve $\Delta \mathrm{C}^{*}$ değerleri ise Tablo 4'te verilmiştir.

Trumpet mantarından elde edilen doğal boya ile boyanmış ipek örneklerinde; en yüksek toplam renk farklılık değeri, bakır sülfat mordanının kullanıldı̆̆ı boyamada elde edilmiştir $\left(\Delta \mathrm{E}^{*}=11,1287\right)$. Trumpet mantarına ait bütün boyamalar standarttan daha açık, daha mat ve mavi nüanslıdır. Kırmızı- yeşil ekseni için ise bakır sülfat mordanın kullanıldığı boyama hariç, diğerlerinde renk yeşil nüanslıdır (Tablo 4).

Tablo 4. Trumpet (Craterellus tubaeformis) Mantarından Elde Edilen Doğal Boya ile Boyanmış Örneklerin $\Delta E^{*}, \Delta L^{*}, \Delta a^{*}$, $\Delta b^{*}$ ve $\Delta C^{*}$ De ğerleri

\begin{tabular}{l|c|c|c|c|c}
\hline Adı & $\Delta E^{*}$ & $\Delta L^{*}$ & $\Delta a^{*}$ & $\Delta b^{*}$ & $\Delta C^{*}$ \\
\hline $\mathrm{CuSO}$ & 11,1287 & 10,19 & 0,91 & $-4,38$ & $-4,31$ \\
\hline $\mathrm{KAl}_{4}\left(\mathrm{SO}_{4}\right)_{2}$ & 3,9999 & 3,36 & $-0,21$ & $-2,16$ & $-2,18$ \\
\hline $\mathrm{K}_{2} \mathrm{Cr}_{2} \mathrm{O}_{7}$ & 1,2866 & 1,11 & $-0,03$ & $-0,65$ & $-0,65$ \\
\hline $\mathrm{SnCl}_{2}$ & 3,8301 & 2,39 & $-0,37$ & $-2,97$ & $-2,99$ \\
\hline $\mathrm{FeCl}_{2}$ & 9,0056 & 6,85 & $-1,3$ & $-5,7$ & $-5,83$ \\
\hline $\mathrm{FeCl}_{3}$ & 4,3587 & 3,88 & $-0,61$ & $-1,89$ & $-1,96$ \\
\hline
\end{tabular}

\subsubsection{Renk Kuvveti Değerleri}

Boyamaların 400-700 nm dalga boyunda, 10'ar nanometre aralıkla ölçülen reflektans değerleri belirlenerek, minimum absorsiyona ait, boyamanın renk kuvveti değeri denklem 2'ye göre hesaplanmıştır (Tablo 5).

Tablo 5. Chantarelle (Cantharellus Cibarius), Porcini (Boletus Edulis) ve Trumpet (Craterellus Tubaeformis) Mantarlartndan Elde Edilen Doğal Boya ile Boyanmış Örneklerin Minimum Absorbsiyondaki (400nm) Renk Kuvveti (K/S) Değerleri

\begin{tabular}{l|c|c|c}
\hline Adı & Chantarelle & Porcini & Trumpet \\
\hline Mordansiz & 0,2348 & 0,4656 & 0,2412 \\
\hline $\mathrm{CuSO}$ & 0,7498 & 0,7880 & 0,8464 \\
\hline $\mathrm{KAl}_{4}\left(\mathrm{SO}_{4}\right)_{2}$ & 0,2499 & 0,4106 & 0,3859 \\
\hline $\mathrm{K}_{2} \mathrm{Cr}_{2} \mathrm{O}_{7}$ & 0,2128 & 0,3417 & 0,2959 \\
\hline $\mathrm{SnCl}_{2}$ & 0,2896 & 0,5021 & 0,3838 \\
\hline $\mathrm{FeCl}_{2}$ & 0,5362 & 0,9367 & 0,7119 \\
\hline $\mathrm{FeCl}_{3}$ & 0,3976 & 0,5030 & 0,4202 \\
\hline
\end{tabular}


Ayrıca Tablo 5 yardımı ile oluşturulan boyamaların renk kuvveti grafikleri Şekil 5'de verilmiştir.

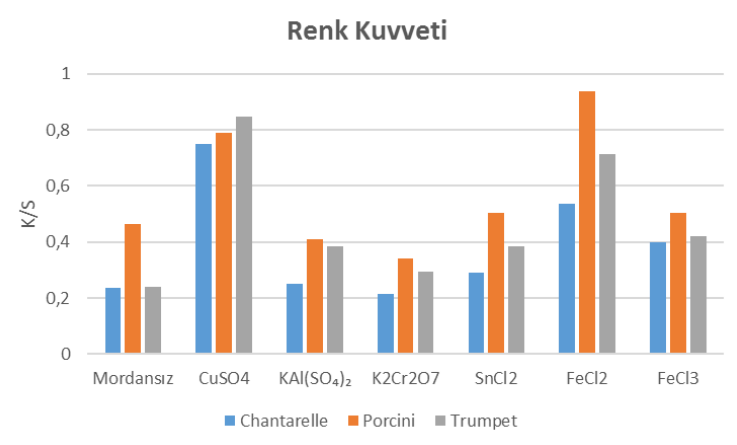

Şekil 5. Chantarelle (Cantharellus Cibarius), Porcini (Boletus Edulis) ve Trumpet (Craterellus Tubaeformis) Mantarları ile Boyanmış Örneklerin Minimum Absorbsiyondaki (400nm) Renk Kuvveti (K/S) Grafikleri
Chantarelle, porcini ve trumpet mantarlarından elde edilen boyarmaddeler ile ipekli materyalin boyanmasında renk kuvveti $(\mathrm{K} / \mathrm{S})$ değerleri karşılaştırıldığında; en iyi renk veriminin demir (II) klorür ile mordanlandıktan sonra porcini mantarından elde edilen doğal boya ile boyanmış ipek materyalde elde edildiği görülmektedir. Mordansız boyama, potasyum alüminyum sülfat, potasyum dikromat, kalay(II)klorür ve demir(III)klorür mordanları ile yapılan tüm boyamalarda renk verimi oldukça düşüktür.

\subsubsection{Chantarelle (Cantharellus Cibarius), Porcini (Boletus Edulis) ve Trumpet (Craterellus tubaeformis) Mantarlarından Elde Edilen Doğal Boya ile Boyanmış İpek İpliklerinin Haslık Testleri}

Çalışmada kullanılan mantar ekstraktı boyamalarından elde edilen 1 şı, yıkama ve sürtme haslık değerleri, Chantarelle (Cantharellus Cibarius) Tablo 6'da, Porcini (Boletus Edulis) Tablo 7'de ve Trumpet (Craterellus Tubaeformis) Tablo 8'de verilmiştir.

Tablo 6. Chantarelle (Cantharellus Cibarius) Mantar Ekstraktı ile Boyanmış Örneklerin Renk Haslıkları

\begin{tabular}{l|c|c|c|c|c|c|c|c|c|c}
\hline & Ișık & \multicolumn{4}{|c|}{ Yıkama } & \multicolumn{3}{c}{ Sürtme } \\
\hline & & Renk Değișimi & \multicolumn{3}{|c|}{ Kekeleme } & Kuru & Yaş \\
\hline Mordanlar & & & CA & CO & PA & PET & PAN & WO & & \\
\hline Mordansız & 3 & 3 & $3 / 4$ & $3 / 4$ & 4 & 4 & $3 / 4$ & 4 & $3 / 4$ & 3 \\
\hline Bakır sülfat & 3 & 3 & $3 / 4$ & 3 & 4 & $3 / 4$ & $3 / 4$ & 4 & 4 & $3 / 4$ \\
\hline Potasyum Al sülfat & 3 & $3 / 4$ & 4 & $3 / 4$ & 4 & 4 & $3 / 4$ & $4 / 5$ & 4 & 4 \\
\hline Potasyum dikromat & 3 & 3 & 4 & $3 / 4$ & $3 / 4$ & $3 / 4$ & $3 / 4$ & $4 / 5$ & 4 & $3 / 4$ \\
\hline Kalay klorür & 3 & 3 & $3 / 4$ & 4 & $3 / 4$ & 4 & 4 & $4 / 5$ & 4 & $3 / 4$ \\
\hline Demir (II) klorür & 3 & 3 & 4 & 4 & 4 & $3 / 4$ & 4 & 4 & 4 & $3 / 4$ \\
\hline Demir (III) klorür & 3 & 3 & $3 / 4$ & $3 / 4$ & 4 & $3 / 4$ & $3 / 4$ & $3-4$ & 4 & $3 / 4$ \\
\hline
\end{tabular}

Tablo 7. Porcini (Boletus Edulis) Mantar Ekstraktı ile Boyanmış Örneklerin Renk Haslıkları

\begin{tabular}{|c|c|c|c|c|c|c|c|c|c|c|}
\hline & \multirow[t]{3}{*}{ Işık } & \multicolumn{7}{|c|}{ Yıkama } & \multicolumn{2}{|c|}{ Sürtme } \\
\hline & & Renk Değişimi & & & Le & leme & & & Kuru & Yaş \\
\hline Mordanlar & & & $\mathrm{CA}$ & $\mathrm{CO}$ & PA & PET & PAN & $\mathrm{WO}$ & & \\
\hline Mordans1z & 3 & 3 & $3 / 4$ & $3 / 4$ & 4 & 4 & $3 / 4$ & 4 & 4 & $3 / 4$ \\
\hline Bakır sülfat & 3 & 3 & $3 / 4$ & $3 / 4$ & 4 & $3 / 4$ & $3 / 4$ & 4 & 4 & $3 / 4$ \\
\hline Potasyum Al sülfat & 3 & $3 / 4$ & 4 & $3 / 4$ & $3 / 4$ & 4 & $3 / 4$ & 4 & 4 & $4-5$ \\
\hline Potasyum dikromat & 3 & 3 & $3 / 4$ & $3 / 4$ & 4 & $3 / 4$ & $3 / 4$ & $4 / 5$ & 4 & $3 / 4$ \\
\hline Kalay klorür & 3 & 3 & 4 & 4 & 4 & $3 / 4$ & $3 / 4$ & $4 / 5$ & 4 & $4-5$ \\
\hline Demir (II) klorür & 3 & $3 / 4$ & 4 & 4 & 4 & $3 / 4$ & 4 & 4 & $3 / 4$ & $3 / 4$ \\
\hline Demir (III) klorür & 3 & 3 & $3 / 4$ & $3 / 4$ & 4 & $3 / 4$ & $3 / 4$ & $3-4$ & $3 / 4$ & $3 / 4$ \\
\hline
\end{tabular}

Tablo 8. Trumpet (Craterellus Tubaeformis) Mantar Ekstraktı ile Boyanmış Örneklerin Renk Haslıkları

\begin{tabular}{|c|c|c|c|c|c|c|c|c|c|c|}
\hline \multirow[b]{3}{*}{ Mordanlar } & \multirow[t]{3}{*}{ Işı } & \multicolumn{7}{|c|}{ Yıkama } & \multicolumn{2}{|c|}{ Sürtme } \\
\hline & & \multirow[t]{2}{*}{ Renk Değişimi } & \multicolumn{6}{|c|}{ Lekleme } & Kuru & Yaş \\
\hline & & & $\mathrm{CA}$ & $\mathrm{CO}$ & PA & PET & PAN & WO & & \\
\hline Mordansiz & 3 & 3 & $3 / 4$ & $3 / 4$ & $3 / 4$ & 4 & $3 / 4$ & 4 & 4 & $3 / 4$ \\
\hline Bakır sülfat & 3 & 3 & $3 / 4$ & $3 / 4$ & 4 & $3 / 4$ & 4 & 4 & 4 & $3 / 4$ \\
\hline Potasyum Al sülfat & 3 & $3 / 4$ & 4 & 4 & $3 / 4$ & 4 & $3 / 4$ & 4 & 4 & 3 \\
\hline Potasyum dikromat & 3 & 3 & $3 / 4$ & $3 / 4$ & 4 & $3 / 4$ & 4 & $4 / 5$ & $3 / 4$ & $3 / 4$ \\
\hline Kalay klorür & 3 & 3 & 4 & 4 & $3 / 4$ & 4 & $3 / 4$ & $4 / 5$ & 4 & $3 / 4$ \\
\hline Demir (II) klorür & 3 & $3 / 4$ & $3 / 4$ & $3 / 4$ & 4 & $3 / 4$ & 4 & 4 & 4 & 3 \\
\hline Demir (III) klorür & 3 & 3 & $3 / 4$ & 4 & 4 & $3 / 4$ & $3 / 4$ & $3-4$ & 4 & $3 / 4$ \\
\hline
\end{tabular}


Bolu ili Mengen ilçesinden toplanmış olan chantarelle (Cantharellus Cibarius), porcini (Boletus Edulis) ve trumpet (Craterellus Tubaeformis) cinsi, zehirli olmayan mantarların, ipek materyalin boyanmasında, doğal boya kaynağı olarak araştırıldığ 1 bu çalışmada, bakır sülfat, potasyum alüminyum sülfat, potasyum dikromat, kalay (II) klorür, demir (II) klorür ve demir (III) klorür olmak üzere altı farklı mordanla yapılan boyamaların haslık değerleri orta derecededir (3-4). Bilindiği üzere doğal boyaların haslık özellikleri düşüktür. Haslık özelliklerini iyileştirmek için mordanlama işlemi yapılmaktadır (Zuber ve ark. 2020). Mordan madde açısından haslık özellikleri kıyaslandığında, mordan madde türünün numunelerin haslık özelliklerini çok fazla etkilemediği görülmüştür.

\section{Sonuç}

Doğal boya olarak chantarelle (Cantharellus Cibarius), porcini (Boletus edulis) ve trumpet (Craterellus tubaeformis) yabani mantarların ekstraksiyonun kullanıldığı bu çalışmada ipek kumaşın farklı mordan maddelerle boyanması incelenmiştir. Boyamadan önce ipek iplikler bakır sülfat, potasyum alüminyum sülfat, potasyum dikromat, kalay 2 klorür, demir 2 klorür ve demir 3 klorür ile ön mordanlama işlemine tabi tutulmuştur. Boyama işleminden sonra numunelerin spektrofotometrik ölçümleri ve

\section{Kaynakça}

Ahmad, W. Y. W., Noor, N. Md., Ahmad, M. R., Ab Kadir. M. I., (2014). Microwave-Assisted Extraction as a Rapid Extraction to Produce Natural Dyes from Pycnoporus sanguineus Mushroom. Proceedings of the International Colloquium in Textile Engineering, Fashion, Apparel and Design 2014 (ICTEFAD 2014) pp 95-98.

Anonim 1. Cantharellus cibarius Fr.;

https://www.amazon.com/Chanterelle-Mushroom-Cantharelluscibarius-Mycelium/dp/B0122VBTA4 (erişim tarihi 05.02.2020)

Anonim 2. Boletus edulis Bull. :

Fr.;https://www.mondofunghi.com/boletus-edulis-funghi-

porcini.html (erişim tarihi 25.01.2020)

Anonim 3. Craterellus tubaeformis;

https://commons.wikimedia.org/wiki/File:Trompetenpfifferling Craterellus_tubaeformis.jpg (erişim tarihi 15.01.2020)

Anonim 4.

https:/en.wikipedia.org/wiki/Melanin\#/media/File:Melanin.svg (erişim tarihi 25.07.2019)

Anonim 5. http://www.food-info.net/tr/caro/stru.htm_(erişim tarihi 02.02.2020)

Benli, H., Bahtiyari, M.I. (2015). Combination of ozone and ultrasound in pretreatment of cotton fabrics prior to natural dyeing, Journal of Cleaner Production, 89,116-124.

Bessete, A., Bessete AR. (2001). The rainbow beneath my feet: A mushroom dyer's field guide, Syracuse: Syracuse University Press, pp. 118.

Bhatti, I. A., Adeel, S., Jamal, M. A., Safdar, M., Abbas, M. (2010). Influence of gamma radiation on the colour strength and fastness properties of fabric using turmeric (Curcuma longa L.) as natural dye. Radiat. Phys. Chem., 79, 622-625. haslık özellikleri araştırılmıştır. Sonuçlara göre, mordan maddelerinin, boyamaların CIELAB ve renk koyulukları üzerinde etkili olduğu görülmüştür. Ayrıca mordan çeşidinin haslıklar üzerinde kayde değer bir etkisinin olmadığı sonucuna varılmıştır. Üç farklı mantardan elde edilmiş doğal boyarmaddelerle boyanmış ipekli materyallerin, boyama ve haslık özelliklerine metal mordanların etkilerinin incelendiği bu çalışma, farklı mordanlar ve farklı tekstil materyelleri ile genişletilebilir. Konvansiyonel yöntem dışında çevre dostu boyama yöntemleride kullanılabilir.

\section{Bilgilendirme}

$\mathrm{Bu}$ çalışma Bolu Abant İzzet Baysal Üniversitesi tarafından 2014.18.06.740 numaralı bap projesi ile desteklenmiştir.

Cheung, L.M., Cheung, P.C.K., Ooi, V.E.C. (2003). Antioxidant activity and total phenolics of edible mushroom extracts. Food Chemistry 81, 249-255.

Dayioglu, H.;Kut, D., Merdan, N., Canbolat, Ş. (2015). The Effect of Dyeing Properties of Fixing Agent and Plasma treatment on Silk Fabric Dyed with Natural Dye Extract Obtained from Sambucus Ebulus L. Plant. Procedia - Social and Behavioral Sciences, 195, $1609-1617$.

Ebrahimzadeh, M.A., Safdari, Y., Khalili, M. (2015). Antioxidant Activity of Different Fractions of Methanolic Extract of the Golden Chanterelle Mushroom Cantharellus cibarius (Higher Basidiomycetes) from Iran. International Journal of Medicinal Mushrooms. v17.i6.pages. 557-565.

Elshemy, N.S. (2011). Unconventional Natural Dyeing Using Microwave Heating with Cochineal as Natural Dyes, Research Journal of Textile and Apparel, 15, 4, 26-36.

Hynninen, P.H., Räisänen, R.,Elovaara, P. (2000). Preparative Isolation of Anthraquinones from the Fungus Dermocybe sanguine Using Enzymatic Hydrolysis by the Endogenous $\beta$ GIucosidase. Zeitschrift fur Naturforschung - Section C Journal of Biosciences Volume 55, Issue 7-8, Pages 600-610.

Karuppan P., Sekarenthiran, S. C., Velusamy K., Sadasivam M., Mahalingam P., Ramayanam, B. M. ; Devi S. (2014). Prospective Aspects of Myco-Chrome As Promising Future Textiles. Proceedings of the 8th International Conference on Mushroom Biology and Mushroom Products (ICMBMP8) 2014..s. 412-416.

Kim, J.H., Sung, N.Y., Kwon, S.K., Srinivasan, P., Song, B.S., Choi, J.I., Yoon, Y., Jin Kyu Kim, J.K., Byun, M.W., Kim, M.R., Lee, J.W. (2009). Gamma-irradiation improves the color and antioxidant properties of Chaga mushroom (Inonotus obliquus) extract. J Med Food. 12(6):1343-7.

Kozarski, M., Klaus, A., Jakovljevic, D., Todorovic, N., Vunduk, J., Petrović, P., Niksic, M., Miroslav M Vrvic, M.M., Leo van Griensven, L.V. (2015). Antioxidants of Edible Mushrooms. Oct. 27; 20(10): 19489-19525. 
Lee, Y.H., Kim, H.D. (2004). Dyeing properties and colour fastness of cotton and silk fabrics dyed with Cassia tora L. extract. Fiber. Polym. 5, 303-308.

Liu, M., Zhao, H., Wu, J., Xiong, X., Zheng, L. (2018). Ecofriendly curcumin-based dyes for supercritical carbon dioxide natural fabric dyeing, Journal of Cleaner Production, 197,12621267.

Mirjalili, M., Nazarpoor, K., Karimi, L. (2011). Extraction and identification of dye from walnut green husks for silk dyeing. Asian J. Chem. 23, 1055-1059.

Mongkholrattanasit, R., Krystufek, J., Wiener, J., Vikova, M. (2011). Dyeing, fastness, and UV protection properties of silk and wool fabrics dyed with eucalyptus leaf extract by the exhaustion process. Fibres Text. East. Eur. 19, 3, 94-99.

Mongkholrattanasit, R., Krystufek, J., Wiener, J., Vikova, M. (2011). UV protection properties of silk fabric dyed with eucalyptus leaf extract. The Journal of The Textile Institute . Volume 102, 2011 - Issue 3. Pages 272-279.

Nakpathom, M., Somboon, B., Narumol, N., Mongkholrattanasit, R. (2018). Dyeing of Cationized Cotton with Natural Colorant from Purple Corncob, Journal of Natural Fibers, 15, 5, 668-679.

Nasiry, D., Khalatbary, A.R., Ebrahimzadeh, M.A. (2017).AntiInflammatory and Wound-Healing Potential of Golden Chanterelle Mushroom, Cantharellus cibarius (Agaricomycetes). International Journal of Medicinal Mushrooms, , 19(10):893-903

Perumal K. (2004). Isolation, extraction and dyeing performance of fungal pigment from Ganoderma applanatum, Coriolus versicolor and Amanita muscaria. J. Mycol. Pl. Pathol. 34: 690691.

Prabhu, K.H., Teli, M.D., Waghmare, N.G. ( 2011). Eco-friendly dyeing using natural mordant extracted from Emblica officinalis G. Fruit on cotton and silk fabrics with antibacterial activity. Fiber. Polym. 12, 753-759.

Tayade, P.B., Adivarekar, R.V. (2013). Adsorption kinetics and thermodynamic study of Cuminum cyminum L. dyeing on silk. Journal of Environmental Chemical Engineering. Volume 1, Issue 4, Pages 1336-1340.

Prusty, A.K., Das, T., Nayak, A., Das, N.B. (2010). Colourimetric analysis and antimicrobial study of natural dyes and dyed silk. J. Clean. Prod. 18, 1750-1756.

Rangel-Castro JI., Staffas A., Danell E., (2002) The ergocalciferol content of dried pigmented and albino Cantharellus cibarius fruit bodies, Mycol. Res. 106 (1): 70-73

Rather L.J., Shahid I. ul, Khan M.A., Mohammad F.. (2016) Adsorption and kineticstudies of Adhatoda vasica natural dye onto woolen yarn with evaluations ofcolorimetric and fluorescence characteristics, J. Environ. Chem. Eng. 4 (2) 1780 1796.

Raisanen R. (2009) Dyes from lichens and mushrooms. In: Bechtold B, Mussak R (eds) Handbook of natural colorants. Wiley, UK, pp 183-200.
Raisanen R, Nousiainen P, Hynninen PH (2001) Emodin and dermocybin natural anthraquinones as a high temperature disperse dye for polyester and polyamide. Text Res J 71:922-927.

Raisanen R, Nousiainen P, Hynninen P.H. (2001) Emodin and dermocybin natural anthraquinones as mordant dye for wool and polyamide. Text Res J 71:1016-1022.

Räisänen R. (2002). Anthraquinones from fungus Dermocybe Sanguinea as textile dyes. Academic Dissertation, University of Helsinki, Finland

Rice M.C. and Beebee D. (1980). Mushrooms for color. Eureka, CA: Mad River Press; pp. 153.

Santis, D.D., Moresi, M. (2007). Production of alizarin extracts from Rubia tinctorumand assessment of their dyeing properties, Industrial Crops and Products 26, 151-162.

Sarıkaya, A. G. (2019). Sulu Çözeltilerden Cr(VI)'nın Biyosorpsiyonunda Pleurotus ostreatus'un Biyokütle Olarak Kullanımının Araştırılması. Avrupa Bilim ve Teknoloji Dergisi, (17), 1173-1183.

Shahid, M., Islam,S., Mohammad, F. ( 2013). Recent advancements in natural dye applications: A review. Journal of Cleaner Production 53,310-331.

Tidke, G., Rai, M.K. (2006). Biotechnological Potential of Mushrooms: Drugs and Dye Production. International Journal of Medicinal Mushrooms. Volume 8, Issue 4, pages 351-360

Vankar, P.S., Shanker,R., Verma, A. (2007). Enzymatic natural dyeing of cotton and silk fabrics, without metal mordants, Journal of Cleaner Production 15, 1441-1450.

Vankar, P.S., Shanker, R. ( 2006). Sonicator dyeing of cotton and silk fabric by Ixora coccinea. Asian Text. J., 77-80.

Wang, L., Wang, N., Jia, S., Zhou, Q. ( 2009). Research on dyeing and ultraviolet protection of silk fabric using vegetable dyes extracted from Flos Sophorae. Text. Res. J. 79, 1402-1409.

Weber, R.W., Mucci, A., Davoli, P. (2004). Laetiporic acid, a new polyene pigment from the wood-rotting basidiomycete Laetiporus sulphureus (Polyporales, Fungi). Tetrahedron Lett. 45: 10751078.

Zuber, M., Adeel, S., Rehman, F., Anjum, F., Muneer, M., Abdullah, M., Zia, K.M.(2020). Influence of microwave radiation on dyeing of bio-mordanted silk fabric using neem bark (azadirachta indica)-based tannin natural dye, Journal of Natural Fibers, Volum 17, No 10, p. 1410-1422

ISO 105-C06, Test for Colour Fastness of Textiles-Colour Fastness to Washing.

ISO 105-X12:2016, Tests for Colour Fastness -Part X12: Colour Fastness to Rubbing.

EN ISO 105-B02-1994 Colour fastness to artificial light: Xenon Arc Fading Lamp Test 
Avrupa Bilim ve Teknoloji Dergisi 